\title{
Magnetic Resonance Imaging of the Patellofemoral Joint
}

\author{
Berna DiRIM METE ${ }^{1}$, Merve GÜRSOY ${ }^{1}$, Hikmet KOÇYiĞiT² \\ ${ }^{1}$ Clinic of Radiology, Izmir Atatürk Training and Research Hospital, Izmir, Turkey \\ ${ }^{2}$ Clinic of Physical Medicine and Rehabilitation, Izmir Atatürk Training and Research Hospital, Izmir, Turkey
}

\begin{abstract}
Patellofemoral pain is common in both athletic and nonathletic individuals. It is observed in 15\%-33\% of the young active group and approximately in $21 \%-45 \%$ in adolescence. Among athletes, men tend to present with more patellofemoral injuries than women. In the nonathletic population, women and young individuals more commonly present with patellar disorders. Many static and dynamic internal derangements of the patellofemoral joint in these patients lead to various secondary imaging findings. Because of the excellent contrast resolution and the ability to directly obtain images in three planes, magnetic resonance imaging is the preferred technique for evaluating cartilage abnormalities, instabilities, and contiguous soft tissue injuries of the patellofemoral joint. This review defines the magnetic resonance imaging of common abnormalities of the patellofemoral joint. Chondromalacia patellae can be diagnosed with high accuracy using magnetic resonance imaging. Abnormalities of the patellofemoral alignment can be grossly assessed on static images; however, more sophisticated evaluation of patellofemoral tracking requires the kinematic technique. Traumatic injuries of the patellofemoral joint, such as traumatic patellar dislocation, traumatic injuries of quadriceps, and patellar tendons, are also reviewed.

Keywords: MRI, patellofemoral, instability, chondromalacia patellae, dislocation
\end{abstract}

Patellofemoral pain syndrome is a condition that is often caused by patellar instability, chondromalacia patellae, or patellofemoral malalignment, and it is often observed in individuals younger than 45 years. When magnetic resonance imaging (MRI) of patients with pain, particularly in the anterior or anterolateral part of the knee, is performed, the patellofemoral joint must be evaluated in terms of patellofemoral instability and early cartilage loss. In addition, the ligament structures around the joint must be evaluated using MRI to identify traumatic patellofemoral dislocation requiring surgery and the symptoms that accompany it. Moreover, it is essential to understand the MRI criteria of instability and the anatomy and variations of the joint to make a correct assessment (1).

\section{Patellofemoral Joint Anatomy}

The patellofemoral joint is formed by the articulation of the patella bone and the intercondylar fossa, which is also called the trochlear groove (TG), in the anterior of the femur. The patellar quadriceps, which is the largest sesamoid bone in the body, is embedded in the femoris tendon. The lower pole of the ovalshaped patella is called the apex (2).

While the deep fibrils of the patellar tendon, which are an elongation of the central part of the quadriceps tendon, connect the patella apex with the tuberositas tibia, the superficial layer of this tendon continues with the quadriceps tendon. Neighbor fibrils, forming the medial and lateral patellar retinaculum, extend to the lateral and medial regions by the bone edge of the patella. The patellar retinaculum, through local thickening, 
forms stronger support structures that are called the reticular or patellar ligament complexes (3).

The joint surface of the patella is oval and is separated into the lateral and medial regions with a vertical eminentia. An additional, smaller vertical eminentia in the medial region limits a narrow space called the odd facet in the utmost medial region of the medial joint surface. The thickest cartilage in the knee joint at the patella is $6-7 \mathrm{~mm}$, whereas the thinnest cartilage is $2 \mathrm{~mm}$ at some parts of the tibial plateau (4).

The upper $1 / 3$ of the patella posterior surface is articulated with the femoral TG; however, according to the position of the knee, different patellar articular surfaces are in contact with different areas of the femur. In full flexion, the most medial facet of the patella makes contact with the lateral segment of the medial femoral condyle, whereas the superior segment of the lateral patella facet makes contact with the anterior of the lateral condyle. While the medial facet of the patella moves closer to the lower part of the femoral patellar surface when the knee is in extension, the lowest part of the patella articular facet makes contact with the femur in full extension. When the knee is forced to extension, the patella tends to laterally shift. The distinctive lateral patellar surface of the femur and the supportive muscle and ligament groups in the neighborhood of the patellofemoral joint prevent this shift (3).

The medial and lateral patellar ligament complexes have a very important role in protecting the patellofemoral joint stability during complex movements of the knee. These ligament complexes, which are formed by the condensation of retinacula, bind the patella to the surrounding structures. The medial retinaculum includes the medial patellofemoral ligament (MPFL), medial patella meniscal ligament, and medial patellotibial ligament. MPFL is the largest part of the medial patellar ligament complex, and studies reveal that it plays the greatest role in preventing lateral displacement of the patella $(5,6)$. The horizontal course of the distal fibrils of the vastus medialis muscle, which is also called the vastus medialis obliquus (VMO), is an important protector of the patella in its normal position. A part of the superficial fibrils of MPFL interpenetrates with fibrils of the VMO muscle. In the attachment level of MPFL to the patella, this ligament and fibrils of the $\mathrm{VMO}$ muscle move in a parallel course and cause bilaminar views in sections of the axial MRI (Figure 1). Immediately inferior to this level and more posterior to the medial level of the knee, the crural fascia moves parallel to and outside these two structures and causes a trilaminar view (Figure 2). MPFL is attached to the femoral epicondyle that is right in the neighborhood of the adhesion level of the adductor magnus tendon to the femur (Figure 3). While in $60 \%$ of the population, the superficial fibrils of MPFL directly cling to the bone in the posterior of the tibial collateral ligament (TCL), the deep fibrils combine with TCL (Figure 4). All the fibrils of MPFL in $40 \%$ of patients are attached to TCL (Figure 5) (7).

The medial patellotibial and medial patella meniscal ligaments are the other medial ligament stabilizers of the patellofemoral joint. MPFL, leaving the medial side of the patella, obliquely stretches to the inferior level and ends by connecting to the anterior horn of the medial meniscus and coronary ligament.
This ligament is evaluated in the axial and sagittal MRI (Figure 6). The medial patellotibial ligament attaches to the medial side of the tibia just at the inferior level of the joint. At this level, some fibrils of the medial patellotibial ligament intertwine with TCL. The ligament attaches to the medial side at the inferior of the patellar bone and at the proximal side of the patellar tendon. The medial patellotibial ligament is best displayed in axial MRI directly taken from the inferior of the tibiofemoral joint level (Figure 6).

The lateral patellar retinaculum comprises a lateral patellofemoral ligament at the superior level, which is similar to the medial patellar retinaculum, and comprises the lateral patellomeniscal and patellotibial ligaments at the inferior level. The other lateral supporters of the joint are the patellar extension of the fascia lata and the superficial oblique band of the lateral retinaculum, extending to the patella from the iliotibial band.
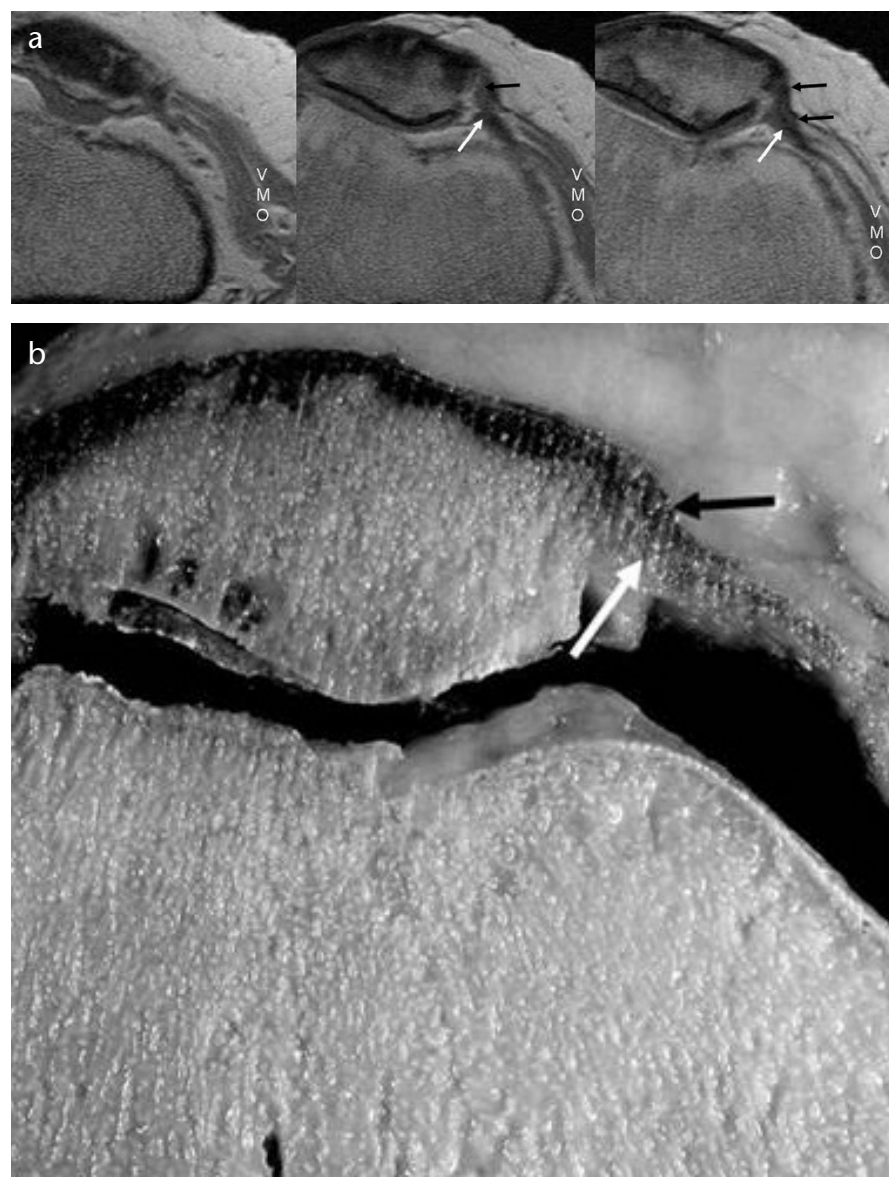

Figure 1. a, b. Normal anatomy of the superior region of the medial patellofemoral ligament complex. (a) In the axial plane of sequential SE T1-weighted MRI sections and (b) in the anatomical sectional image obtained from the knee section of the cadaver, the superior region of the medial patellofemoral ligament (white arrow) and the bilaminar view formed by the parallel course of the vastus medialis obliquus muscle and inferior tendon fibrils (black arrow) are observed. VMO: vastus medialis obliquus muscle 


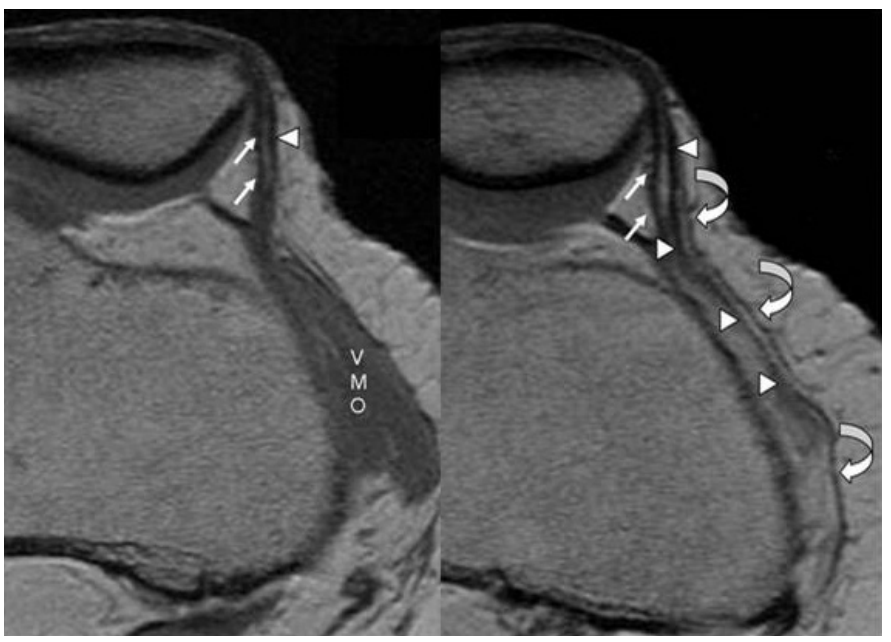

Figure 2. Trilaminar view formed by the medial ligament complex with the crural fascia. In the posterior plane, the crural fascia (inclined arrows), external tendinous fibrils of the vastus medialis obliquus muscle (arrow heads), and superior fibrils of the medial patellofemoral ligament (arrows) are observed in sequential SE T1-weighted MR images

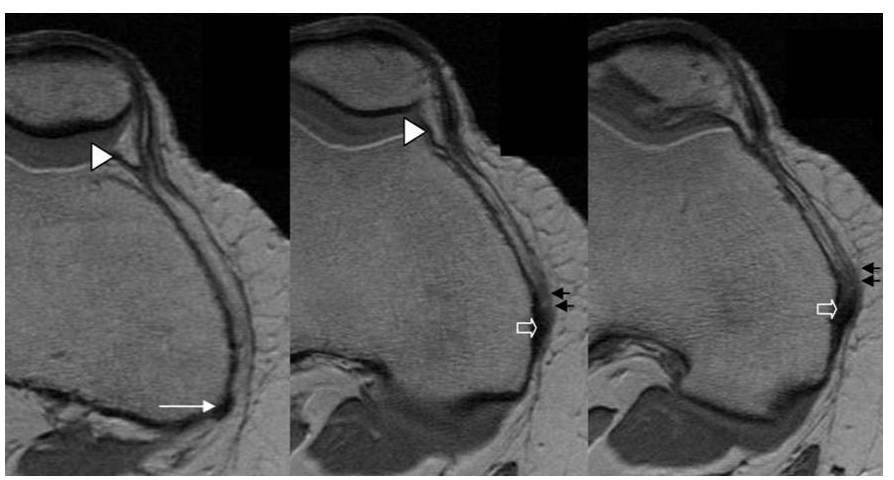

Figure 3. Normal sectional anatomy of the adhesion level of the medial patellofemoral ligament to the femur. In the axial plane from the superior to the inferior region, in sequential SE T1weighted MR images, it is observed that the medial patellofemoral ligament (black arrow) and the tibial collateral ligament (open arrow) are attached to the femoral epicondyle just in the inferior of the adhesion level of the adductor magnus tendon to the femur (white arrow). Arrow heads show the normal synovium of the joint, which is not expanded with fluid

The lateral ligament complex is stronger than the medial complex $(1,6)$.

There are three separate fat pads around the patella: Hoffa's fat pad, quadriceps fat pad, and prefemoral fat pad. The most common fat pad abnormality observed in patellofemoral joint diseases is an edema at the superolateral section of Hoffa's fat pad (1).

Magnetic Resonance Imaging Technique of the Patellofemoral Joint

MRI is the preferred method in revealing the damages of the knee extensor mechanism because of its high soft tissue resoluti-
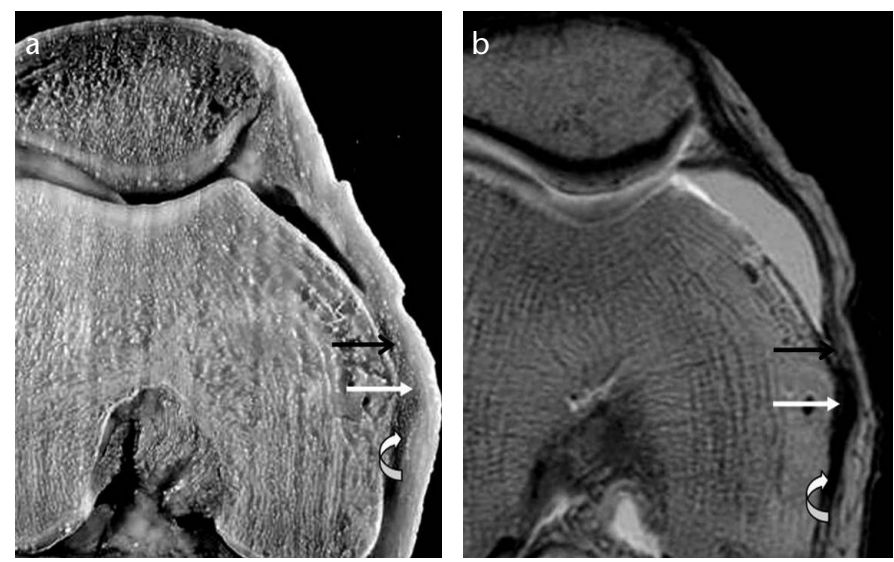

Figure 4. a, b. Normal sectional anatomy of the adhesion level of the medial patellofemoral ligament to the femur. (a) In the axial plane of an SE T1-weighted MR arthrography section and (b) in the anatomical sectional image obtained from the knee section of the cadaver, it is observed that the internal fibrils of the medial patellofemoral ligament (arrowheads) are attached to the tibial collateral ligament (white arrows), and the external fibrils (inclined arrow) are attached to the posterior femur by passing over the tibial collateral ligament
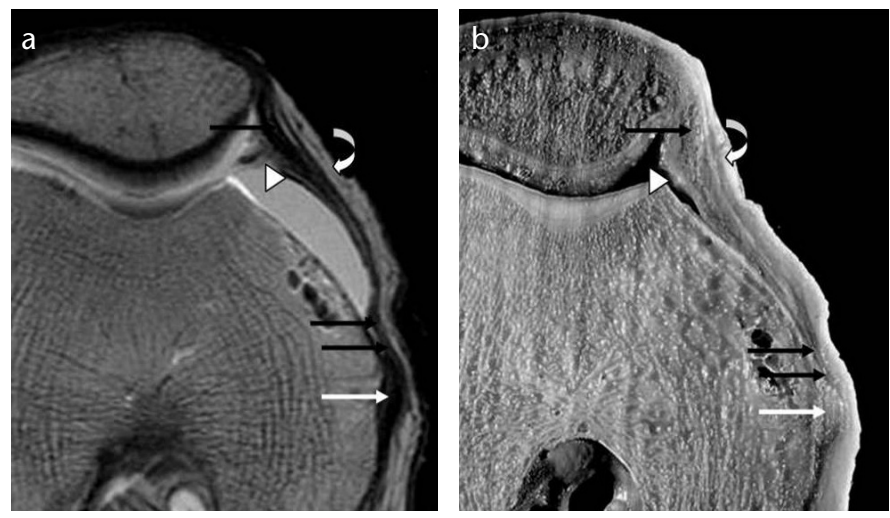

Figure 5. a, b. Normal sectional anatomy of the adhesion level of the medial patellofemoral ligament to the femur. (a) In the axial plane of a SE T1-weighted MR arthrography section and (b) in the anatomical sectional image obtained from the knee section of the cadaver, the fibrils of the medial patellofemoral ligament (black arrow) intertwine with the tibial collateral ligament (white arrow); they attach to the femur in this way. The inclined arrow shows the crural fascia, and the arrowhead shows the joint synovium

on and multiplanar capability. Special extremity coils and a small anterior surface coil can be used to obtain high-resolution scans in the limited area of the patellofemoral joint in some cases.

The display area in the sagittal images should include the area from the rectus femoris muscle tendon intersection right up to the inferior region of the tibial tuberosity (TT).

While examining the patellofemoral joint, routine MRI protocol should include sagittal spin-echo T1, sagittal fatsuppressed fast spin echo T2/proton density (PD) or short tau inversion recovery, axial fat-suppressed fast spin echo (FSE) 


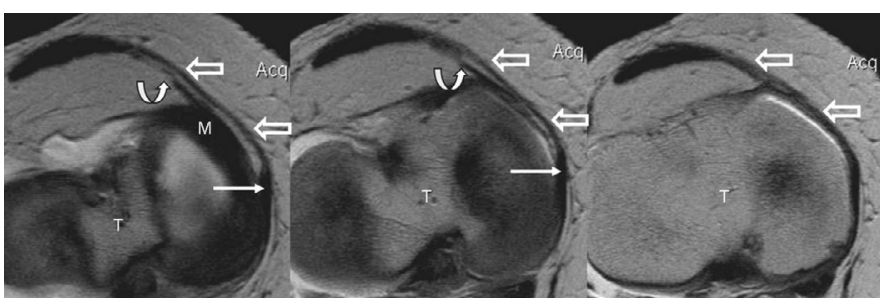

Figure 6. Normal sectional anatomy of the medial patellomeniscal and medial patellotibial ligaments. In the axial plane of sequential SE T1-weighted MRI arthrography sections, the medial patellomeniscal ligament (inclined arrow), medial patellotibial ligament (open arrow) and tibial collateral ligament (white arrow) are observed. M: anterior horn of the medial meniscus, T: tibia

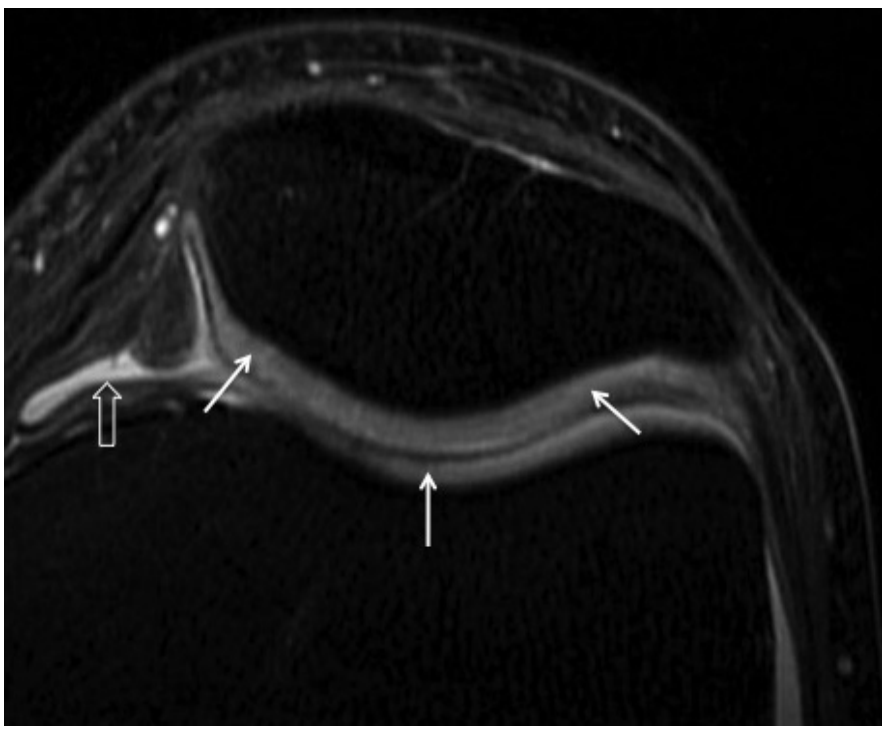

Figure 7. Normal joint cartilage. In the fat-suppressed intermediate axial MR section, the femoral trochlear groove cartilage (white arrows) is observed as uniform and with mildly lower signal, compared to the cartilage fluid (open arrow), with lateral and medial patellar facets. Cartilage and bone can be differentiated at the optimum level

proton density, and 60 sagittal spoiled gradient echo (SPGR) (spoiled GRASS (gradient recalled acquisition in the steady state) with 3-mm section thickness and 0-mm section intervals for the evaluation of joint cartilage or specific sequences to provide a similar cartilage evaluation. To eliminate the pulsation artifacts that may result from popliteal artery phase encoding, the direction should be from the left to right and not from the front to back.

Sequences, such as spin echo (SE), gradient echo (GRE), FSE, and three-dimensional (3D) SE and GRE, that are used in cartilage imaging with MRI enable morphological assessment. Recent developments in hardware, software, gradient coils, and radiofrequency have led to improvements in FSE (TSE), the use of fat-suppressed and fluid-exciting sequences, and consequently, improvements in tissue contrast. While the cartilage and bone can be well differentiated in T1, SE, and FSE sequences, these

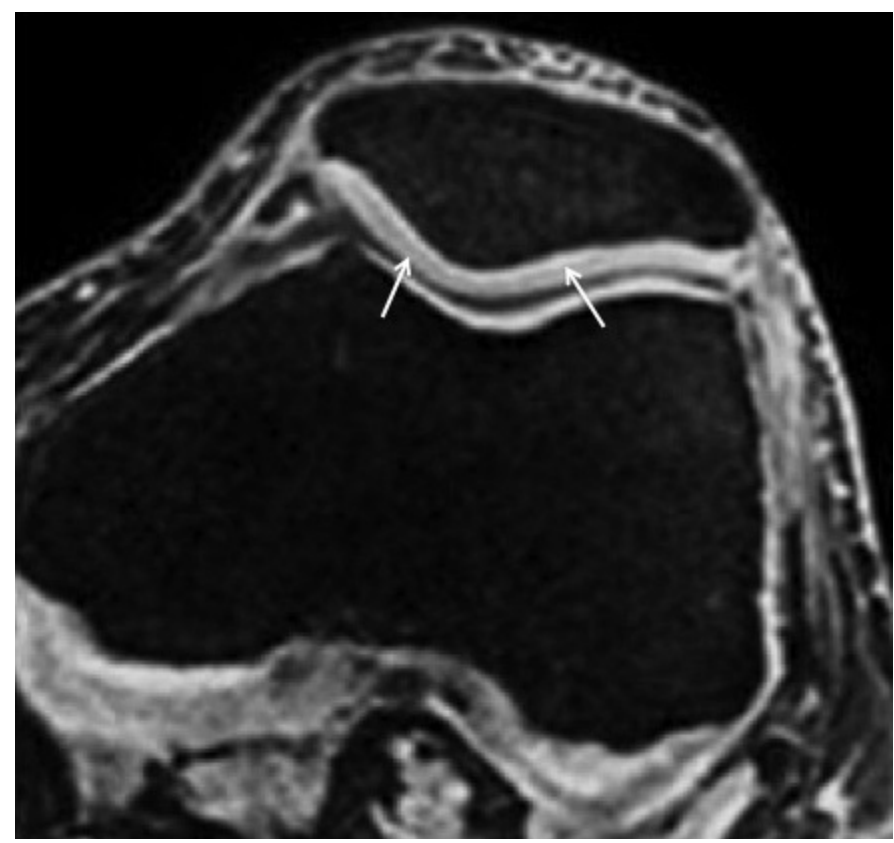

Figure 8. In the fat-suppressed 3D SPGR sequence, on the axial plane, a low-signal band that divides the patellar cartilage into two parts from the midline level is observed in accordance with the truncation artifact (white arrows)

techniques have low contrast in separating the cartilage and liquid (Figure 7). In addition, they are not effective in identifying signal changes in the early stages of cartilage pathology. The contrast between the cartilage and liquid is high in the conventional T2-SE sequence. However, the signal-view ratio is insufficient and is not effective in imaging the early stages of cartilage pathology (8). Fat-suppressed FSE, T2, PD, and 3D-SPGR are defined as the sequences providing the most accurate information in the evaluation of articular cartilage; these sequences can be used as a routine $(4,9)$. Fat-suppressed 3D-spoiled gradient recalled echo (3D-SPGR) imaging is quite successful in assessing the size and structural integrity of the cartilage tissue (9). 3D-SPGR enables the display of the cartilage with a high signal in the neighborhood of the synovial fluid with a low signal and good resolution. Fissures and losses of the cartilage are easily identified. Articular cartilage is observed as a high-band signal in this sequence. A low-signal line passing through the midline of the high-signal cartilage is frequently monitored in this sequence. This view is attributed to a truncation artifact [an MRI artifact that develops if the signals (sampling) cannot be recorded in the time required for imaging in Fourier transformation] (Figure 8).

Fourier transform (DEFT) imaging (unlike conventional MRI, strengthening the signal from the synovial fluid creates more liquid contrast) is effective for imaging the whole cartilage thickness. In 3D-dual echo steady state (in which the signal density of the joint fluid is very high, and the cartilage tissue can be distinguished if there is enough visible joint fluid), both the cartilage and synovial fluid have a high signal. High signal-view ratio, near-isotropic sections, and short imaging times are the advantages of this examination. T2 mapping enables the imaging of 


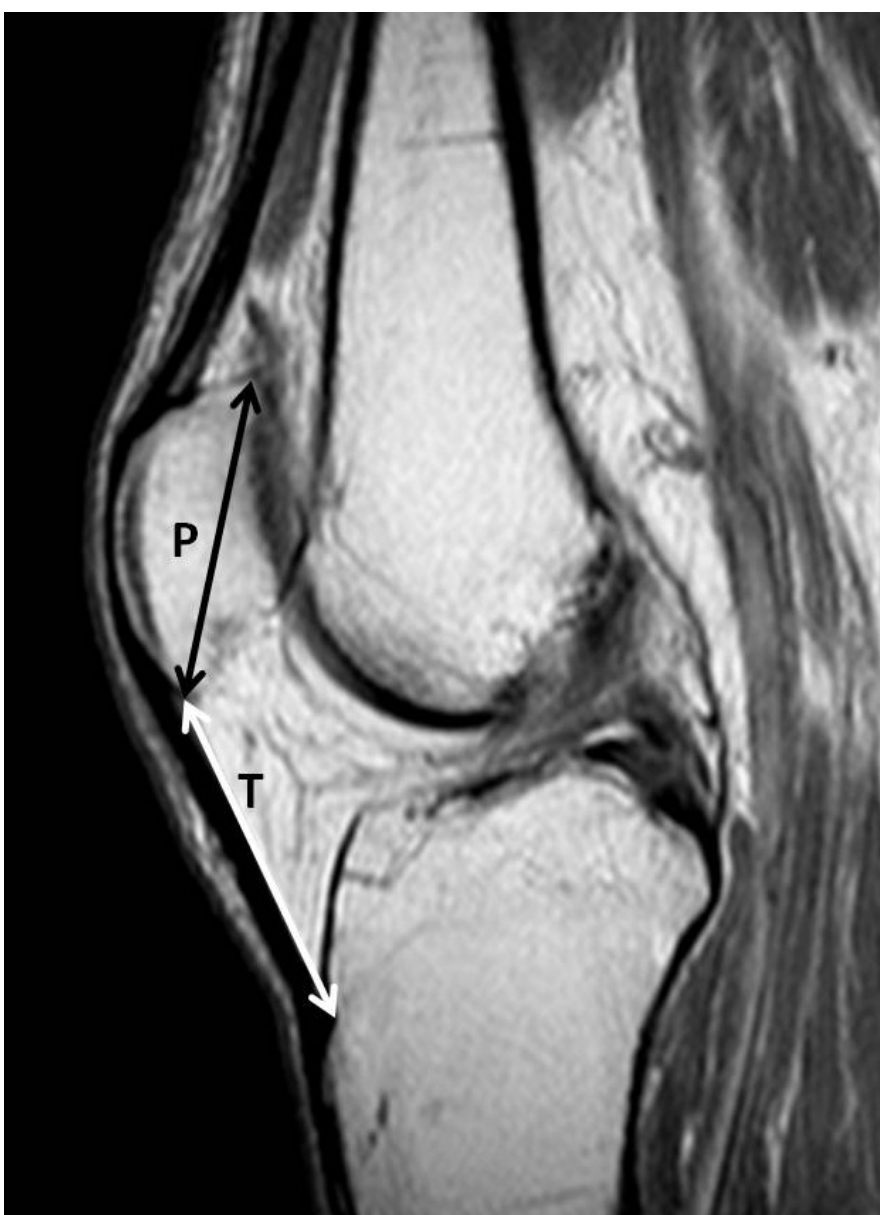

Figure 9. Normal patellar position. In the sagittal plane, in a proton density MR section from the midsagittal level, when the length of the shortest line (white arrow) drawn parallel to the deepest edge of the patellar tendon $(\mathrm{T})$ is proportioned to the distance (black arrow) between the antero-inferior and postero-superior corners of the patella $(P)$, the values ranging from 0.75 to 1.3 demonstrate the patella to be in a normal position

the cartilage by delayed-gadolinium MRI bright display; moreover, physiological imaging techniques, such as T1rho mapping, sodium MRI, and diffusion-weighted imaging, enable the evaluation of the molecular composition of the cartilage. New MRI techniques are being developed to assist in understanding the process of cartilage destruction.

In direct imaging of cartilage lesions, MR arthrography (MRA) is used in selected patients. Fat-suppressed SE sequences are used for imaging the cartilage in this technique. In the literature, $100 \%$ specificity is reported for the MR-A technique (10). Direct MR-A is the most effective method for the staging of osteochondral lesions, and it should be used before surgery (11). Indirect MR-A is recommended when invasive intervention is contraindicated or in the absence of technical information and fluoroscopy for direct MR-A (11).

Some special kinematic MRI techniques can be exploited to investigate the dynamic displacement of the patellofemoral joint (12).

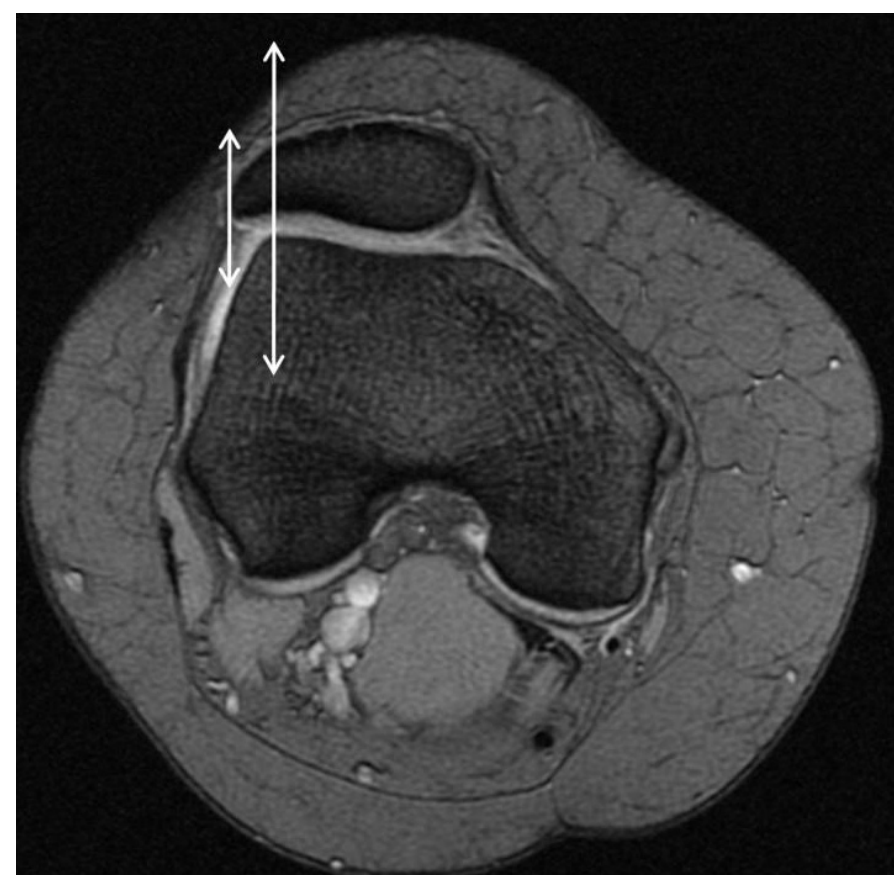

Figure 10. Lateral patellar subluxation. In the axial plane of a fatsuppressed SE T2-weighted MR section, the distance between the line drawn perpendicularly to the medial corner of the patella (short arrow) and the line drawn perpendicularly to the most anterior segment of the medial femoral condyle (long arrow) is observed to be $>5 \mathrm{~mm}$

\section{Magnetic Resonance Imaging in Patellofemoral Joint Pat- hologies}

Patella shape variations and the patellar position (patella alta and baja) should be defined, and articular cartilage damage, soft tissue structures of the medial and lateral patellar retinaculum in the neighborhood of the joint, fat pads, quadriceps and patellar tendon pathologies, patellar tilt (using the patellofemoral angle), and TG hypoplasia/dysplasia (using the TG angle) should be investigated while patellofemoral joint pathologies are evaluated using MRI $(1,3)$.

\section{-Patellar Shape Variations}

Many variations of the patellar joint surface of the patella and femur were defined. Some of these variations cause patellar instability and retropatellar pain. Many different patellar shape classifications are available; the Wiberg classification is the most commonly used (13). Three basic patellar configurations were defined in this classification. Type I has concave lateral and medial patellar facets of equal size. In type II patella, which is the most common, the joint surfaces are concave, but the medial facet is slightly smaller than the lateral facet. Type III patella was defined as a minor medial facet and convex articular surface (3). It is considered that the Wiberg type III patella, wherein the lateral facet is prominent and the medial facet is small, is among the predisposing factors of patellar instability and chondromalacia; however, this has not been proven $(3,14)$. 


\section{-Patellofemoral Malalignment and Instability}

Rotational deviation of the patella related to any axis is called patellar malalignment (15). Patellar instability is an entity that is characterized by a shift, subluxation, or dislocation of the patella (3).

Lateral shift of the patella is prevented by the support of passive and active stabilizers. Passive and active stabilizers of the patella are the quadriceps and patellar tendon, medial and lateral patellar retinaculum, three medial and three lateral reticular ligaments, and mainly, the VMO muscle around the patella. The passive medial stabilizers are MPFL, medial patella meniscal ligament, and medial patellotibial ligament; these three structures play a primary role in stabilizing the knee, particularly at $20^{\circ}-30^{\circ}$ of the flexion. MPFL is regarded as the most powerful passive medial stabilizer to prevent the lateral slipping of the patella (57). The identification of MPFL lesions using MRI will enable these lesions to be properly repaired. The medial patellomeniscal and patellotibial ligaments have less importance in stabilization (7). The most important active medial stabilizer is the VMO muscle. While the oblique fibers of the $\mathrm{VMO}$ muscle adhere to the patella with an average angle of $55^{\circ}$, the vastus lateralis fibers adhere at an average angle of $14^{\circ}$. The fibrils of the VMO muscle create resistance against the vastus lateralis to pull the patella to a lateral position during the extension of the knee and prevent the bone from laterally slipping. Before the trochlea becomes a part of the activity, at the very beginning of the flexion, the VMO muscle plays an important role in preventing lateral patellar subluxation (6). Because the distal fibrils of the VMO muscle often hold the adductor magnus tendon, the adductor magnus muscle also contributes to stabilization.

Different factors, such as developmental or acquired abnormalities affecting patellar shape and position or the size and shape of TG, pathologies of adjacent muscle groups, ligament laxity, and traumatic damage to passive and active stabilizers of the patella, can cause patellar instability (15). While investigating patellofemoral malalignment and instability, MRI examinations of the knee should be evaluated in terms of the position of the patella, patellar tilt and subluxation, trochlear inclination, and dysplasia.

\section{-Evaluation of Patellar Position: Patella Alta and Baja}

In routine MRI examination that are obtained when the knee is extended, in the midsagittal section, a 1/3 segment of the lower patella must articulate with the femoral trochlear surface. A higher than normal position of the patella is called patella alta, while a lower than normal position is called patella baja. Patella alta is associated with repeated patellar subluxation or dislocation, and chondromalacia patella is associated with Sinding-Larsen-Johansson disease and joint effusion $(3,16)$. Patella baja is associated with achondroplasia and neuromuscular diseases.

Many different methods have been defined to diagnose patella alta and baja by lateral radiograph $(1,3)$. The Insall-Salvati (IS) ratio is the method most commonly used to assess the patellar position; it is calculated by dividing the patellar tendon length by the maximum diagonal length of the patellar bone in a knee radiograph. The normal value of this ratio is $1.02 \pm 0.13$; if a standard deviation of 2 points more is observed, the diagnosis is patella alta, whereas if the standard deviation is 2 points less, the diagnosis is patella baja (17).

Because the lengths of the patellar tendon and the patella are different from each other in each of the MRI sections, evaluating this through MR images is more complicated than through lateral radiography. In a study, these measurements were obtained using a single midsagittal image (18). In MRI, the length of the patella is the distance between the anterior-inferior and posterior-superior corners, and the length of the patellar tendon is determined by measuring the shortest line drawn parallel to the deepest edge of the patellar tendon (Figure 9). If the ratio of the tendon length to the patella length is greater than 1.52 in men and 1.32 in women, a patella alta diagnosis is made; if the ratio is smaller than 0.79 in men and 0.74 in women, a patella baja diagnosis is made (18).

\section{-Patellar Tilt and Subluxation}

In MRI and CT, multiple static images taken at different degrees of flexion over axial slices can be observed in the cine format. However, factors, such as a contracted quadriceps muscle and a slightly flexed knee position during the examination may affect the position of the patella. The positions of the patella and TG can be easily assessed in axial slices obtained during extension and minimal flexion in routine MRI.

Patellar subluxation is evaluated through axial MR slices. The distance between the line perpendicularly drawn to the medial corner of the patella and the line perpendicularly drawn to the most anterior segment of the medial femoral condyle is measured (19). Values of $2 \mathrm{~mm}$ or less are considered normal (20), from 2 to $5 \mathrm{~mm}$ are mild subluxation, from 5 to $10 \mathrm{~mm}$ are moderate subluxation, and $10 \mathrm{~mm}$ or higher are severe subluxation (Figure 10). Severe subluxation is more frequently observed in women (1). In another measurement method for the evaluation of patellar subluxation, the distance between the line perpendicularly drawn to the most lateral edge of the medial articular surface of the patella and the line perpendicularly drawn to the apex of TG is measured. These lines should roughly intersect in normal cases.

Abnormal patellar tilt can occur together with patellar subluxation or alone. Patellar tilt to the lateral aspect without subluxation is called excessive lateral pressure syndrome. This syndrome often develops because of the tension in the lateral patellar retinaculum; surgery is performed in patients who do not respond to conservative treatment. Different methods were described for evaluating patellar tilt $(18,21)$. Patellofemoral angle measurement is still a simple and valid method; the angle between the line drawn along the lateral patellar facet and the line drawn tangent to the anterior femoral condyle is evaluated. The mid-point of the patella is determined on the basis of sagittal slices, and the angle is measured at this level. The patellofemoral angle is generally $>8^{\circ}$, and the lines forming the angle are laterally open (Figure 11). Opening towards the medial or an angle $<8^{\circ}$ is considered as an abnormal tilt (1). An alternative measurement was defined by Grelsam et al. (21) and Wittste et al. (22) In this method, the angle between the line passing through the lateral and medial edges of the patella and the line 

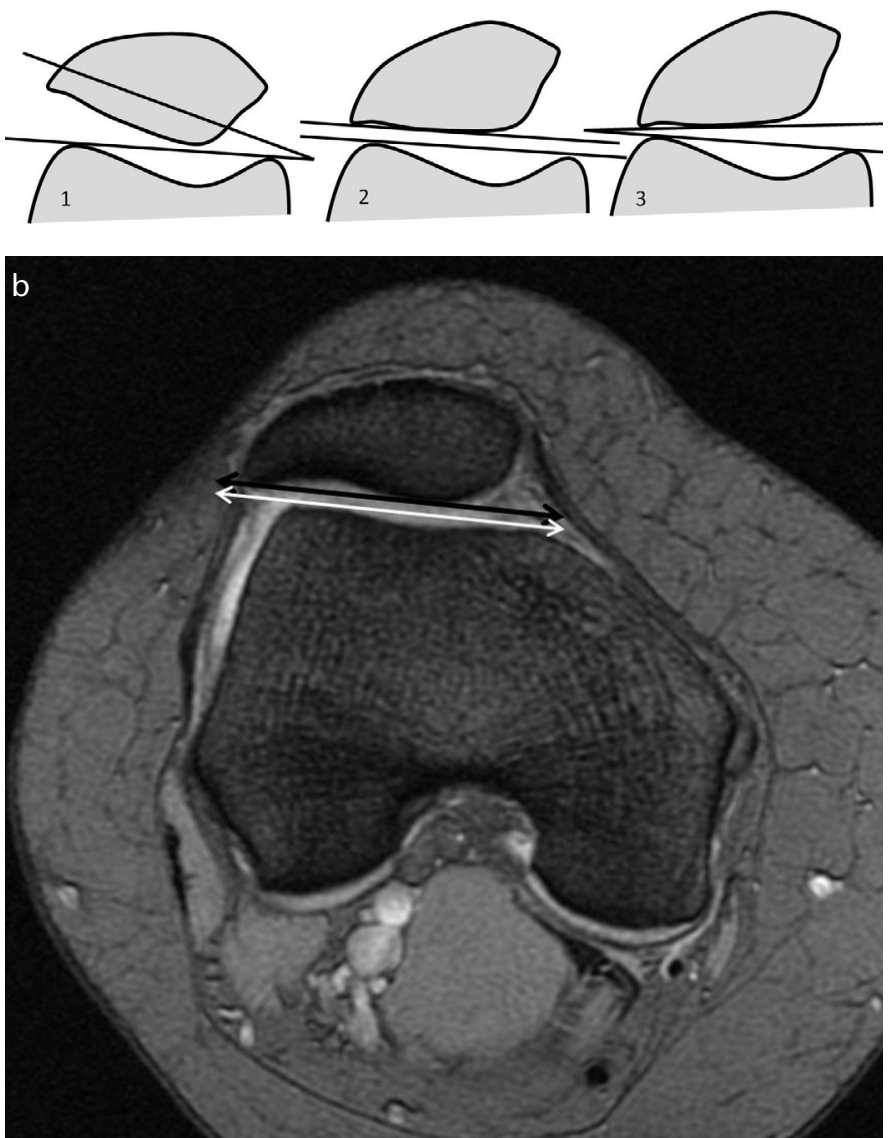

Figure 11. a, b. (a) The evaluation of patellar tilt in an axial MR section. If the patellofemoral angle between the line drawn along the lateral patellar facet and the line drawn tangent to the anterior femoral condyle is greater than 8 degrees and the lines forming the angle are laterally open (1), the patella is in the normal position. If the angles between two lines are parallel to each other (2), medially open (3), or smaller than 8 degrees, the tilt is considered to be abnormal. (b) In the patient with abnormal patellar tilt, the two lines are observed to be parallel to each other
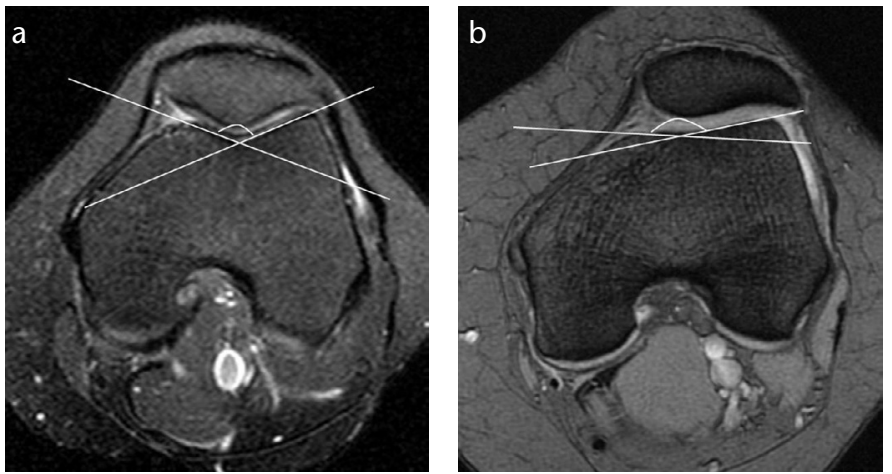

Figure 12. a, b. Trochlear dysplasia. (a) Normal trochlear groove in a SPIR T2-weighted section in the axial plane (groove angle, $<144^{\circ}$ ), and (b) hypoplasic trochlear groove in a fat-suppressed SE T2-weighted section in the axial plane (groove angle, $>144^{\circ}$ ) are observed drawn tangent to the posterior femoral condyle is measured. It was reported that this angle was $\geq 10^{\circ}$ in patients with a distinct patellar tilt in physical examination (21). However, it may be difficult to use this technique because the two lines of the angle to be measured are often on two separate axial MRI slices. When assessing patellar tilt and subluxation through MRI, a point to keep in mind is that the patella often laterally slides when there is a great amount of effusion.

Open and vertical MRI systems provide a more natural assessment of the joint movement. With different gradients recalling imaging methods, speed imaging and motion-triggered cine MRI (cinematographic representation of sequential images taken with knee in motion) enable viewing with the load imposed on the joint by imitating the walk. These latest methods provide us with information regarding excessive lateral pressure syndrome and medial subluxation of the patella, which is a complication of lateral retinacular release surgery $(3,12)$. However, in our daily practice, it is possible to accurately evaluate patellar tilt and subluxation with routine MRI of the knee.

\section{-Evaluation of the Trochlear Inclination and Dysplasia}

Patellar instability and dislocation are often accompanied by the patellar bone or TG dysplasia. A shallow TG or shallow angle of the lateral wall of the groove causes a decrease in the stability of the patellofemoral joint, patellar subluxation, or even a predisposition to the dislocation. In addition, osteochondroma at the TG level or nonunion of a femoral fracture can be acquired causes of impaired TG morphology.

Trochlear analysis has been attempted in the past by making various measurements from axial radiography images obtained while the knee was flexed at $30^{\circ}$. Currently, this assessment can be made with higher accuracy using axial MR images. Pfirrmann et al. (23) reported that the diagnosis of trochlear dysplasia can be made with $100 \%$ sensitivity and $96 \%$ specificity rate if the trochlear depth is $3 \mathrm{~mm}$ and below in the measurement made from an axial MR slice taken from 3-cm proximal of the tibiofemoral joint line.

Moreover, Carrillo et al. (24) described the parameters of determining lateral TG inclination through axial MRI sections. The most proximal axial section where trochlear cartilage surface is observed is selected as a reference. A line is perpendicularly drawn to the posterior surface of both femoral condyles; the angle between a second line perpendicularly drawn to the trochlear surface of the lateral femoral condyle is measured. This angle describes the lateral trochlear inclination. This angle is $<11^{\circ}$ in patellar instability (sensitivity, $93 \%$; specificity, $87 \%$ ).

The TG angle is the angle between the lines drawn tangent to the medial and lateral facet joint surfaces of the femoral condyles. When measuring the groove angle based on the cartilage, different results can be obtained because the thickness of the cartilage may vary from person to person. Thus, measurements made over bony structures appear to be more reliable. In cases where trochlear dysplasia is suspected, a TG angle of $>144^{\circ}$ indicates hypoplasia (Figure 12) (25). If the depth of the groove is $<5 \mathrm{~mm}$, it is defined as hypoplasia; if it is $<3 \mathrm{~mm}$, it is defined as dysplasia (1). These abnormalities can be treated through trochleoplasty. 

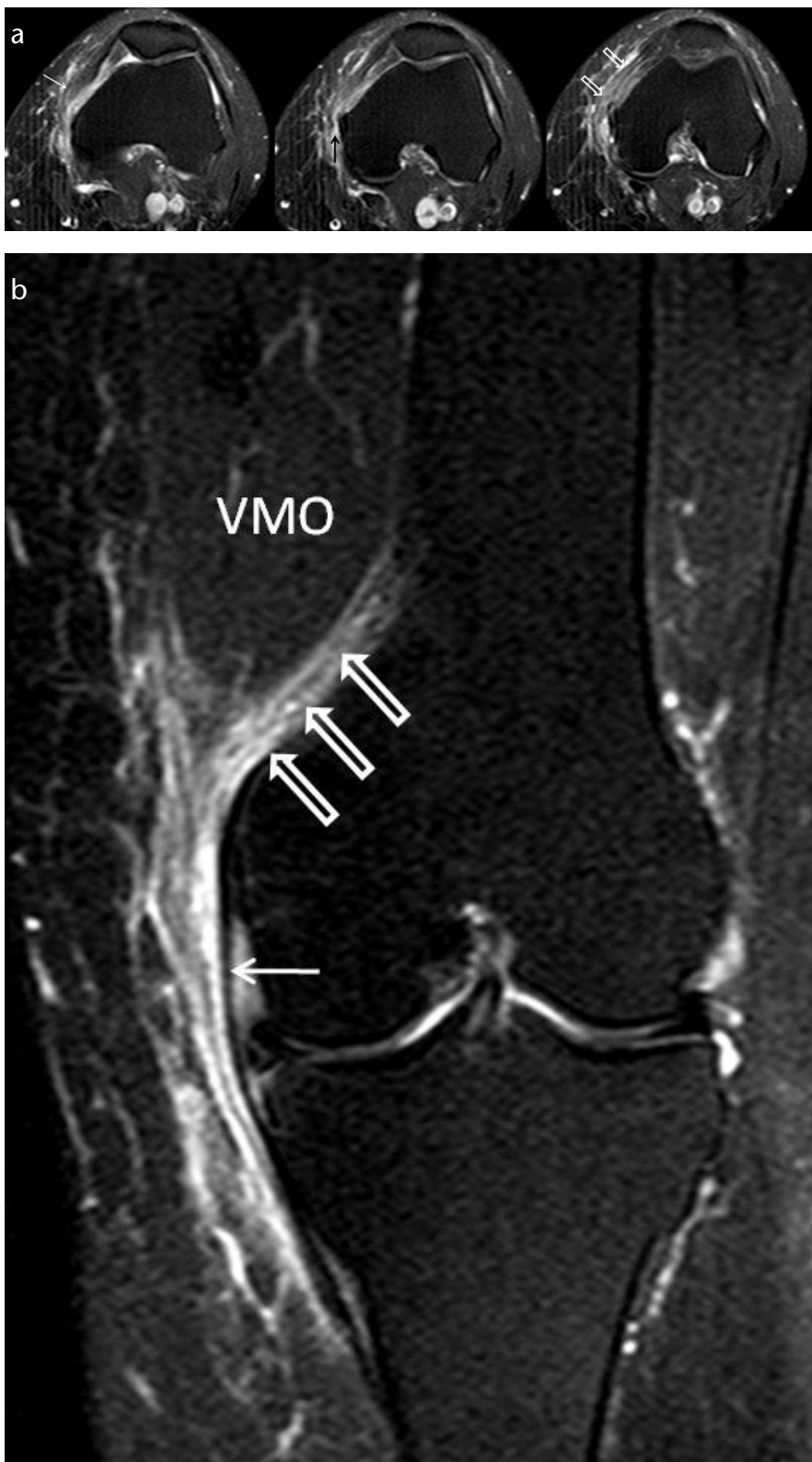

Figure 13. a, b. Rupture at the adhesion level of the medial patellofemoral ligament to the femur (a) In SPIR T2-weighted sequential sections in the axial plane, rupture at the adhesion level of the medial patellofemoral ligament to the femur (black arrow); in the inferior segment of the vastus medialis obliquus muscle, edema-related mild high-signal (white arrow); and in the middle segment of the medial patellofemoral ligament, prominent thickening and high signal consistent with partial damage (blank arrows). (b) In a SPIR T2-weighted section in the coronal plane, edema in the deep region of the vastus medialis obliquus muscle (VMO) and fluid-related high signal (blank arrows) and edema in the superficial neighborhood of the tibial colleteral ligament

\section{-Evaluation of Tibia Tubercle-Trochlear Groove Distance}

Measuring the distance between $T T$ and TG is made using axial slices. This is the maximum distance between the line per- pendicularly drawn to the deepest area of TG and the line perpendicularly drawn to the central level where the TT insertion of the patellar tendon occurs. A TT deviation of $\geq 15 \mathrm{~mm}$ shows lateralization/transposition (1). Patellar tendon/TT lateralization increases lateral traction of the quadriceps femoris muscle onto the patella and creates a predisposition to patellofemoral diseases (26). This lateralization causes friction between the quadriceps tendon and anterior femur and may lead to edema in the superolateral part of the Hoffa's fat pad and the formation of bursa. This situation is also called extreme lateral friction syndrome $(1,3)$. Measuring the TT-TG distance is important for TT osteotomy, particularly for patients in whom re-fixation is planned.

The quadriceps angle ( $Q$ angle), which is measured during the physical examination, is the angle between two lines, the first of which is drawn between the anterior superior iliac crest and the patella center and the second of which is drawn between the patella center and TT. The normal value is considered to be $14^{\circ}\left( \pm 3^{\circ}\right)$ in men and $17^{\circ}\left( \pm 3^{\circ}\right)$ in women. The patella has a tendency toward lateral subluxation in patients with large $Q$ angles. This angle is a good indicator of patellofemoral malalignment. An evaluation similar to the clinical $Q$ angle can be made through sequential coronal MR images. The angle measured between the line passing through the patellar bone midline and the line passing through the center of the tendon at the level where the patellar tendon adheres to TT can be used for this purpose (1).

\section{-Assessing Surrounding Soft Tissues}

Patellofemoral malalignment and instability may be accompanied by edema in the superolateral part of Hoffa/s fat pad, bursa formation in this localization, edema of the prefemoral and quadriceps fat pads, and abnormalities of the trochlear articular cartilage. Edema of the superolateral part of Hoffa's fat pad can be easily identified in distant lateral sagittal sections in fluidsensitive sequences. With time, edema can replace hypertrophy and can cause an enlargement of the fat pad. A fat pad whose size increases may extend under the lateral patellar retinaculum or may create an anterior cambering in the patellar tendon (1).

\section{-Acute Patellar Dislocation and Subluxation}

Acute patellar dislocation often occurs after a single trauma in adults and is usually observed without an underlying predisposing factor in athletes. The most common traumatic mechanism that causes acute patellar dislocation is the internal rotation of the femur with respect to the tibia when the knee is flexed and the foot is in a fixed position. Acute patellar dislocation often develops without a significant trauma in children and adolescents. In these cases, predisposing factors, such as lateral femoral condyle hypoplasia, shallow trochlea, patella alta, vastus medialis muscle hypoplasia, femoral anteversion, medial retinaculum laxity, tense lateral retinaculum, and iliotibial band contracture, exist and may cause patellar malalignment and instability (27). Repeated dislocations are commonly observed after acute dislocation in this group. After repeated patellar dislocation and subluxation, thinning of MPFL and atrophy of the VMO muscle may develop (3). 


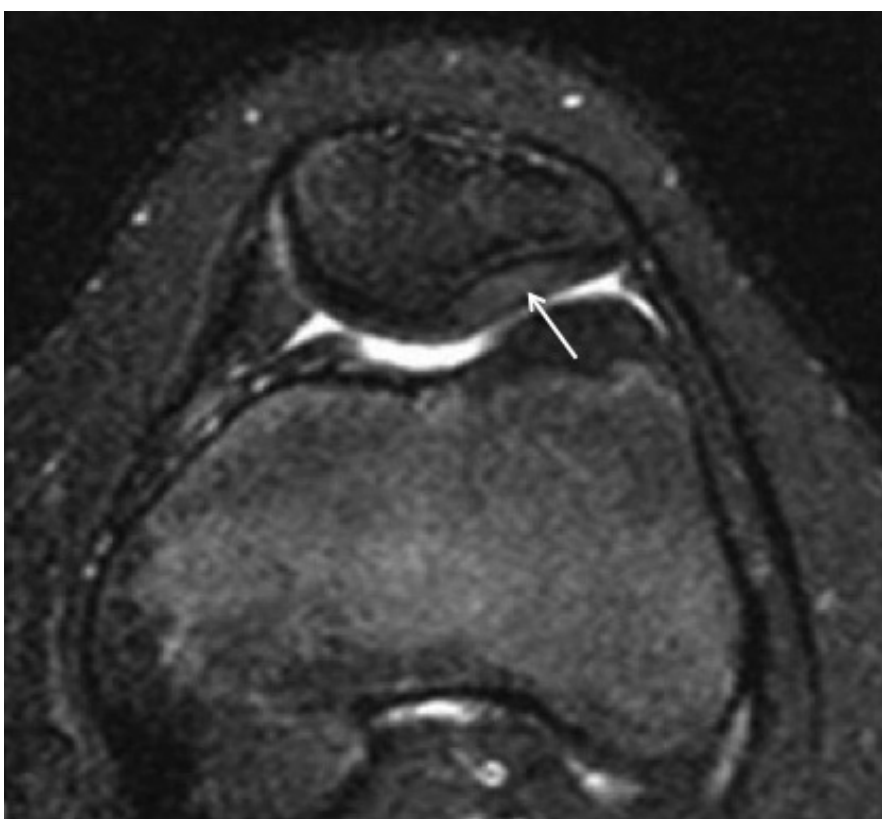

Figure 14. In an axial fat-suppressed proton density-weighted MR image, increased signal consistent with stage 1 chondromalacia patella is observed in the lateral patellar facet of the articular cartilage

Patellar retinaculum and adjacent bone-cartilage damages developing in acute patellar dislocation are defined in detail with MRI.

\section{Patellar Retinaculum Damage}

Medial patellofemoral ligament is considered to be the most powerful passive medial stabilizer that prevents the patella from lateral slipping. After traumatic patellar retinaculum damage, isolated repair of MPFL can provide the patella with lateral stability $(7,27)$. Identifying MPFL lesions through MRI, which are of critical importance in patellar stability, will enable proper repair. The variational absence of this ligament can be observed in a portion of the population; this situation can lead to repeated patellar instability. The medial patellomeniscal and patellotibial ligaments have less importance in stabilization.

Medial patellofemoral ligament damage in patients with acute lateral patellar dislocation is observed at high rates (70\%-100\%). This ligament, which extends between the medial margin of the patella and the immediate anterior of the medial epicondyle of the femur, often breaks off at the sticking level of the femur (Figure 13). Nomura (28) defined that MPFL has a separate pattern of damage in patients with acute lateral patellar dislocation. The first pattern is complete separation of the ligament from the femoral sticking level. The second pattern is an avulsion-type tear where the deep fibrils of MPFL split from the sticking place. The ligament is not ruptured in this type. Tears at the patellar sticking level of the ligament are rarely reported. The superior fibrils of MPFL interpenetrate the deep fascia of the distal portion of the VMO muscle. Therefore, MPFL damage is accompanied by edema and hemorrhage in VMO and accumulation of blood or fluid under this muscle $(3,7)$.

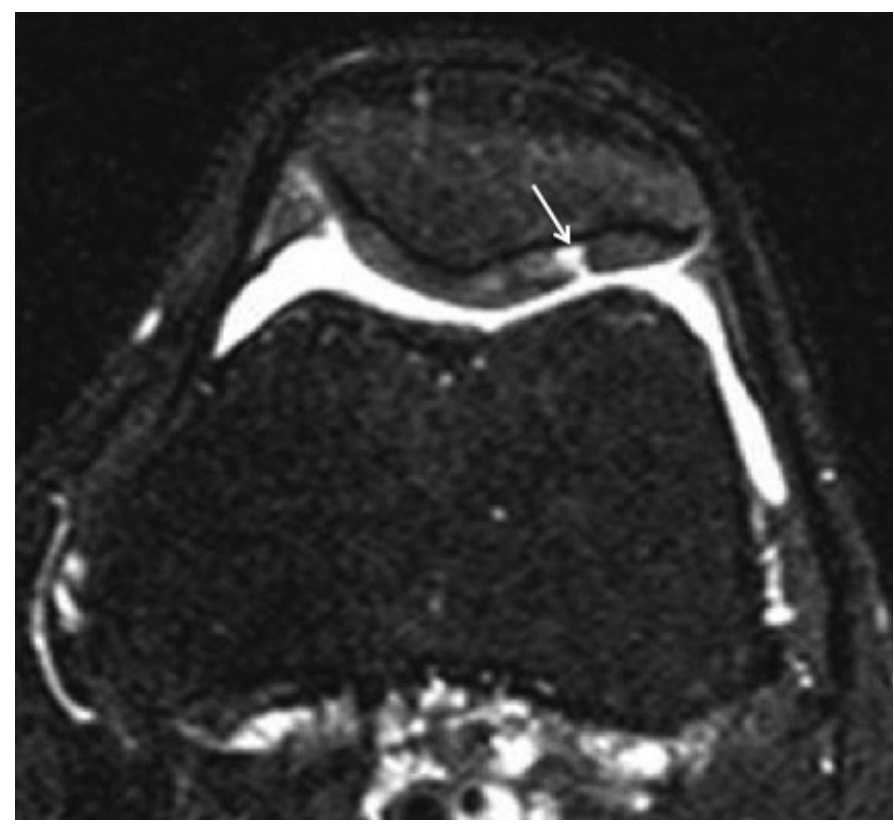

Figure 15. In a fat-suppressed T2-weighted axial MR image, a fullthickness focal defect in the lateral patellar facet of the articular cartilage is observed (white arrow). The extension of joint liquids into the defect provides good imaging of the defect

Many findings accompanying the rupture of MPFL at the femoral sticking level are determined by MRI. Soft tissue edema observed at the femoral sticking level and the superficial neighborhood of the ligament often extends onto the retinacular fibrils towards the anterior and to the superficial neighborhood of TCL towards the posterior. Detachment of the anterior fibrils of TCL from the femur may accompany ligament tear. In MRI slices, retraction in some muscle fibrils, edema, and hemorrhage in deep parts of the muscle are frequently observed at the sticking level of $\mathrm{VMO}$ to the femur. Edema and fluid collection between the distal fibrils of this muscle and the adductor tubercle can cause elevation in the muscle fibrils (29).

\section{Bone and Cartilage Damage}

Damage develops in the lateral femoral condyle and medial patellar facet that are in contact with the dislocated patella when the lateral patellofemoral ligament is in full dislocation. Bone injury, osteochondral fracture, and chondral damage are other pathologies that may develop according to the severity of dislocation. Bone injury and edema in the lateral femoral condyle typically has a peripheral and elongated appearance. Edema is observed extending towards the anterior surface of the condyle in at least one axial section and frequently in more than one section. Aliasing or camber in the lateral condyle surface is an indicator of osteochondral fracture. If the knee undergoes a trauma in the flexed position, probably due to contact of the femoral condyle with the lateral tibial plateau, osteochondral fractures may occur on the lateral femoral condyle middle and posterior surfaces that are exposed to weight. 
Although edema is observed in the medial patellar facet in acute lateral patellar dislocation, the amount and incidence of this edema are less than those of lateral femoral condyle edema. In a study where MRI was performed on patients eight weeks after dislocation, osteocondral damage was reported in $70 \%$ of patients and impaction deformity on the edge of the inferomedial patella in $44 \%$ of patients (30).

\section{Chronic Habitual Patellar Dislocation}

In every flexion of the knee, lateral dislocation of the patella or permanent lateral dislocation of the patella is called chronic habitual patellar dislocation or instability. This situation, frequently seen in children, may develop due to congenital or acquired causes. Idiopathic contraction of the quadriceps muscle, repetitive injections made into the thigh, and trauma affecting the quadriceps muscle may cause this pathology (3).

\section{Chondromalacia Patella}

Cartilage loss affecting one or more sections of the patella and causing patellofemoral pain is called chondromalacia patella. The pathophysiology of this disease is not certainly known. Two distinct mechanisms, base degeneration and surface degeneration, were identified. The base degeneration is the fasciculation of collagen of the middle and deeper layers of the cartilage; it is believed that it is seen in the eminentia between the medial and odd facets of the patella among young people. Excessive pressure is thought to be associated with this condition. The surface degeneration begins in youth and continues during the patient's lifetime. The disease probably ends with patellofemoral joint osteoarthritis (3).

Magnetic resonance imaging is the main research method for displaying the patellofemoral joint cartilage, due to its high resolving power and because it provides the opportunity for multiplanar evaluation $(4,9)$. Although arthroscopic examination is a gold standard for cartilage lesions, some blind areas of arthroscopy, subchondral bone, and all joint structures can be evaluated by MRI; this guides the programming of treatment (31).

Many different classifications have been defined based on the severity of the loss of articular cartilage or its anatomical extent at the chondromalacia patella. In arthroscopic examination, which is the gold standard in the assessment of articular cartilage, a modified MRI staging system based on the Outerbridge arthroscopy staging system can be used in the classification of cartilage defects. In this classification, the evaluation is: Phase 0 , normal cartilage; Phase 1, "softening" or edema without contour irregularities in cartilage (Figure 14); Phase 2, fragmentation in cartilage, fissuring or focal defects below 50\%; Phase 3, 50\% fragmentation in cartilage or below, fissuring or defects; Stage 4, full-thickness cartilage lesions (Figure 15) $(4,31)$.

While a defect in articular cartilage is distinguished by a focal signal reduction in fat-suppressed 3D SPGR sequences, it is distinguished by a focal signal increase in fat-suppressed FSE (TSE) PD-weighted sequences. In addition, areas of local thinning in the cartilage can be evaluated by MRI. The truth value in determining the thinning of the articular cartilage in both techniques is $>90 \%$. If a complete loss exists in the thickness of the cartilage, subchondral bone edema can be evaluated in fat-suppressed
FSE (TSE) PD-weighted sequences, and it is identified in $83 \%$ of patients. Edema in the subchondral bone appears as high intensity signals in this sequence, and intra-articular osteophyte formation is observed in $15 \%$ of patients (4).

Traumatic cartilage lesions usually bypass the cartilage completely and cause signal changes in the subchondral bone. In this case, a signal change in the subchondral bone is a stimulus for cartilage lesions, as described above. MRI findings of osteoarthritis lesions are different. Osteoarthritis lesions are usually observed in large number in MRI and are accompanied by diffuse thinning of cartilage. The size and depth of the defects are variable. The edge of a cartilage defect is generally blunt and shows a wide angulation $(4,31)$.

Although MRI has a high accuracy in showing changes in articular cartilage, false negative results are obtained in delamination injuries, in flap-shaped tears, and in cases where fibrillation occurs in cartilage. As delamination tears, in particular, require surgical treatment, attention should be paid (4).

\section{Evaluation of Quadriceps and Patellar Tendon}

In MRI of the patellofemoral joint, the distal quadriceps tendon, the patellar tendon, the distal vastus lateralis, and pathologies of the medialis muscle should also be evaluated.

If tendinosis, partial and full-thickness tears, or atrophy in muscles are present in tendons, it must be reported. A partial tear in the tendon is recognized by reduction in thickness and high signal; full-thickness tears are recognized with focal absence and frequently with high signal of the fluid in this area. In patients with anterior knee pain, the evaluation of the patellar tendon is important in terms of jumper's knee and OsgoodSchlatter disease (1).

Peer-review: Externally peer-reviewed.

Author Contributions: Concept - B.D.M., M.G., H.K.; Design B.D.M., M.G.;

Supervision - B.D.M., H.K.; Resource - B.D.M., M.G.; Materials B.D.M.; Data Collection and/or Processing - B.D.M., M.G., H.K.; Analysis and/or Interpretation - B.D.M., M.G., H.K.; Literature Review - B.D.M., M.G.; Writer - B.D.M., M.G.; Critical Review - B.D.M., H.K.

Conflict of Interest: No conflict of interest was declared by the authors.

Financial Disclosure: The authors declared that this study has received no financial support.

\section{References}

1. Chhabra A, Subhawong TK, Carrino JA. A systematised MRI approach to evaluating the patellofemoral joint. Skeletal Radiol 2011;40:375-87. [CrossRef]

2. Barnett AJ, Gardner RO, Lankester BJ, Wakeley C], Eldridge JD. Magnetic resonance imaging of the patella: a comparison of the morphology of the patella in normal and dysplastic knees. J Bone Joint Surg $\mathrm{Br}$ 2007;89:761-5. [CrossRef]

3. Resnick D, Kang SK, Pretterkleiber ML. Chapter 25: Knee. In: Resnick D, Kang SK, Pretterkleiber ML, editors. Internal derangements of joints. 2nd ed. Philadelphia: Saunders Elsevier; 2007. p.1561-2011.

4. McCaulay RT, Recht MP, Disler DG. Clinical imaging of articular cartilage in the knee. Sem Musc Skel Rad 2001;5:293-304. [CrossRef] 
5. Amis AA, Firer P, Mountney J, Senavongse W, Thomas NP. Anatomy and biomechanics of the medial patellofemoral ligament: review. Knee 2003;10:215-20. [CrossRef]

6. Panagiotopoulos E, Strzelczyk P, Herrmann M, Scuderi G. Cadaveric study on static medial patellar stabilizers: the dynamizing role of the vastus medialis obliquus on medial patellofemoral ligament. Knee Surg Sports Traumatol Arthrosc 2006;14:7-12. [CrossRef]

7. Dirim B, Haghighi P, Trudell D, Portes G, Resnick D. Medial patellofemoral ligament: cadaveric investigation of anatomy with MRI, MR arthrography, and histologic correlation. AJR Am J Roentgenol 2008;191:490-8. [CrossRef]

8. Cicuttini F, Forbes A, Asbeutah A, Morris K, Stuckey S. Comparison and reproducibility of fast and conventional spoiled gradient-echo magnetic resonance sequences in the determination of knee cartilage volume. J Orthop Res 2000;18:580-4. [CrossRef]

9. Peterfy CG, Schneider E, Nevitt M. The osteoarthritis initiative: report on the design rationale for the magnetic resonance imaging protocol for the knee. Osteoarthritis Cartilage 2008;16:1433-41. [CrossRef]

10. Disler DG, McCauley TR, Wirth CR, Fuchs MD. Detection of knee hyaline cartilage defects using fat-suppressed three-dimensional spoiled gradient-echo MR imaging: comparison with standard MR imaging and correlation with arthroscopy. AJR Am J Roentgenol 1995; 165:377-82. [CrossRef]

11. Imhof $H$, Nöbauer-Huhmann IM, Krestan C, Gahleitner A, Sulzbacher I, Marlovits S, et al. MRI of the cartilage. Eur Radiol 2002;12:2781-93.

12. Shellock FG, Powers CM. Kinematic MRI of the Patellofemoral Joint. Kinematic MRI of the Joints: Functional Anatomy, Kinesiology, and Clinical Applications. CRC press LLC, 2001. [CrossRef]

13. Wiberg G. Roentgenographic and anatomic studies on the femoropatellar joint, with special reference to chondromalacia patellae. Acta Orthop Scand 1941;12:319-410. [CrossRef]

14. Stefancin JJ, Parker RD. First-time traumatic patellar dislocation: a systematic review. Clin Orthop Relat Res 2007;455:93-101. [CrossRef]

15. Grelsamer RP. Patellar malalignment. J Bone Joint Surg Am 2000;82:1639-50.

16. Insall J, Goldberg V, Salvati E. Recurrent dislocation and the highriding patella. Clin Orthop 1972;88:67-9. [CrossRef]

17. Insall J, Salvati E. Patella position in the normal knee joint. Radiology 1971;101:101-4. [CrossRef]
18. Shabshin N, Schweitzer ME, Morrison WB, Parker L. MRI criteria for patella alta and baja. Skeletal Radiol 2004;33:445-50. [CrossRef]

19. Katchburian MV, Bull AM, Shih YF, Heatley FW, Amis AA. Measurement of patellar tracking: assessment and analysis of the literature. Clin Orthop Relat Res 2003;412:241-59. [CrossRef]

20. Biedert RM, Gruhl C. Axial computed tomography of the patellofemoral joint with and without quadriceps contraction. Arch Orthop Trauma Surg 1997;116:77-82. [CrossRef]

21. Grelsamer RP, Weinstein $\mathrm{CH}$, Gould J, Dubey A. Patellar tilt: the physical examination correlates with MR imaging. Knee 2008;15:3-8. [CrossRef]

22. Wittstein JR, Bartlett EC, Easterbrook J, Byrd JC. Magnetic resonance imaging evaluation of patellofemoral malalignment. Arthroscopy 2006;22:643-9. [CrossRef]

23. Pfirrmann CW, Zanetti M, Romero J, Hodler J. Femoral trochlear dysplasia: MR findings. Radiology 2000;216:858-64. [CrossRef]

24. Carrillon Y, Abidi H, Dejour D, Fantino O, Moyen B, Tranh-Minh VA. Patellar instability: assessment on MR images by measuring the lateral trochlear inclination-initial experience. Radiology 2000;216:582-5. [CrossRef]

25. Salzmann G, Weber T, Spang J, Imhoff A, Schöttle P. Comparison of native axial radiographs with axial MR imaging for determination of the trochlear morphology in patients with trochlear dysplasia. Arch Orthop Trauma Surg 2010;130:335-40. [CrossRef]

26. Shakespeare D, Fick D. Patellar instability-can the TT-TG distance be measured clinically? Knee 2005;12:201-4. [CrossRef]

27. Insall JN, Falvo KA, Wise DW. Chondromalacia patellae. J Bone Joint Surg Am 1976;58:1-8.

28. Nomura E. Classification of lesions of the medial patellofemoral ligament in patellar dislocation. Int Orthop 1999;23:260-3. [CrossRef]

29. Sanders TG, Morrison WB, Singleton BA, Miller MD, Cornum KG. Medial patellofemoral ligament injury following acute transient dislocation of the patella: MR findings with surgical correlation in 14 patients. J Comput Assist Tomogr 2001;25:957-62. [CrossRef]

30. Elias DA, White LM, Fithian DC. Acute lateral patellar dislocation at MR imaging: injury patterns of medial patellar soft tissue restraints and osteochondral injuries of the inferomedial patella. Radiology 2002;225:736-43. [CrossRef]

31. Hayes $\mathrm{CH}$, Conway WF. Evaluation of articular cartilage: radiographic and cross-sectional imaging techniques. Radiographics 1992;12:409-28. [CrossRef] 